\title{
Study of Factors Influencing the Length of Hospital Stay of Patients with Anxiety Disorders
}

\author{
Soraya Mohammadi, ${ }^{1}$ Jamshid Yazdani-Charati, ${ }^{2,3,}{ }^{*}$ Mehran Zarghami, ${ }^{3,4}$ Nadia Alipour, ${ }^{1}$ and Afsaneh \\ Fendereski $^{1}$ \\ ${ }^{1}$ Department of Biostatistics, Student Research Committee, School of Health, Mazandaran University of Medical Sciences, Sari, Iran \\ ${ }^{2}$ Department of Biostatistics, School of Health, Health Sciences Research Center, Mazandaran University of Medical Sciences, Sari, Iran \\ ${ }^{3}$ Psychiatry and Behavioral Sciences Research Center, Addiction Institute, Mazandaran University of Medical Sciences, Sari, Iran \\ ${ }^{4}$ Department of Psychiatry, School of Medicine, Mazandaran University of Medical Sciences, Sari, Iran \\ Corresponding author: Jamshid Yazdani-Charati, Department of Biostatistics, School of Health, Health Sciences Research Center, Mazandaran University of Medical Sciences, \\ Sari, Iran. Tel: +98-1133543081-3, Fax: +98-1133542473, E-mail: jamshid.charati@gmail.com
}

Received 2017 March 11; Revised 2017 September 03; Accepted 2017 December 02.

\begin{abstract}
Background: Anxiety disorders are the most common groups of mental disorders. Based on the world health organization reports 7.4\% of global DALYs are caused by disorders, which are in the mental and behavioral category. One of the problems of these patients is the length of stay in the hospital, which can be studied in various aspects.

Objectives: The objective of this study was to determine the effects of related factors on the duration of hospitalization in patients with anxiety disorders.

Methods: A historical cohort of patients placed in the psychiatric center in Sari, northern Iran, were studied from April 2007 to March 2012. Statistical analysis using Weibull regression and stata.12 was performed at the significant level of 0.05.

Results: A total of 427 persons were studied. The median length of hospital stay was 17 (inter quartile range 10 - 29 day). The results showed age was associated with the length of stay $(\mathrm{P}=0.036)$. Also, patients with previous hospitalization and patients who received electro convulsive therapy and occupational therapy had a longer stay in the hospital $(\mathrm{P}<0.001)$.

Conclusions: The overall results showed that the type of treatment is effective in reducing the duration of hospitalization. Aging has a subtractive effect on the length of hospital stay. It seems that additional research concerning mental health care services may be required to identify more factors affecting the length of stay.
\end{abstract}

Keywords: Anxiety Disorders, Length of Stay, Psychiatry, Survival Analysis

\section{Background}

Human beings have always been worried about physical health, social relationships, and his place in the world, and raised many questions and theories in this regard. Since mental disorders are often eccentric or annoying and draws attention, different reactions such as anger, resentment, reluctance, fear, and confusion may be seen. There is later annoyance to the individual or those around him, whether there is a tendency to treat these disorders or not. Some disorders easily affect one's work and social life and often disrupt his performance (1). Anxiety disorders, which were in the category of "Neuroses", in the 18th century, were used for the first time by "William Cullen", a Scottish physician. During the 19th century, based on the biogenesis theory of neuroses, those who had rigidity and self-defeating behavior, despite healthy physical appearance, known as neuroticism, it was thought that they are suffering from unknown "neurological disorders". At the beginning of the 20th century, this theory was replaced by Freud's "Psychogenesis" theory. Freud coined the term "Anxiety Neuroses» and emphasized on the fact that neurotic disorders does not arise from physical causes, but is rooted in anxiety; in the sense when unconscious repressed memories and desires attempt to reach the level of consciousness, anxiety as a warning signal from the base "Ego" becomes apparent and emerges in the form of neurotic disorders. Freud's theory found popularity and become the base of neuroses classification in DSM published by the American psychiatric association (2). "Anxiety" stems from the Latin word "Anxious" in the sense of tightness in the chest. Anxiety refers to the fear of unknown origin, the person does not know why he's afraid, or fear of danger seems inappropriate (3). "Anxiety disorders" includes those mental disorders, which anxiety is the

Copyright (c) 2017, Iranian Journal of Psychiatry and Behavioral Sciences. This is an open-access article distributed under the terms of the Creative Commons Attribution-NonCommercial 4.0 International License (http://creativecommons.org/licenses/by-nc/4.0/) which permits copy and redistribute the material just in noncommercial usages, provided the original work is properly cited 
central point of symptoms of the disorder. In other words, in such disorders symptoms of anxiety are crucial. After mood disorders, the most prevalent psychiatric illnesses are anxiety disorders (4). These types of disorders are usually treated with a combination of biological and psychological therapies. The world health organization (WHO) estimates that $7.4 \%$ of global DALYs are caused by disorders in the mental and behavioral disorders category. Among all disorders, anxiety disorder accounts for $1.08 \%$ of all global DALYs (5). One of the problems of psychiatric patients is duration of hospital stay, which the factors affecting it can be studied in various aspects. Hospitals are the main centers in the health system with crucial roles on the efficacy of health system (6). These health settings consume half of the national expenses in the health sector (7). Demand on the use of hospital services is increasing. One of the ways the governments use for the management of increasing demand of the hospital services is an analysis of the use of hospital services (8). Analysis of the information related to the hospitals performance and rate of benefiting services helps management and decision making of the managers. Hospital stay duration is a main index, which is used extensively nowadays and is one of the simplest indices of hospital activities. This index is used for various objectives, including the hospital care managements, quality central necessity of using hospital services in the hospital management (9). Duration of hospital stay is generally used as an efficiency index. On this aspect, hospital stay duration reduction leads to the increase of efficiency through increase in the number of patients or reduction in number of hospital beds (10). Reduction of unnecessary hospital stay leads to the giving more health services to more number of the patients and reduces the investment pressure and establishment of new health care setting (11). In response to these concerns, this study focused on identifying variables that may be associated with the duration of hospitalization.

\section{Objectives}

This study aimed to determine the affecting factors on the duration of hospitalization in patients with anxiety disorders, using survival analysis methods.

\section{Materials and Methods}

This was a historical cohort study. A total of 2397 medical documentations of patients with psychiatry disorders were studied at a referral psychiatric hospital in Sari, northern Iran from April 2007 to March 2012. According to the information recorded in the documents, 427 patients with anxiety disorder (including generalized anxiety disorder, acute stress disorder, posttraumatic stress disorder, obsessive-compulsive disorder, panic disorders, phobias, etc.) were entered in the study. Data collection tool was a checklist comprising of the length of hospital stay as dependent variable, and gender, age, residence (native, nonnative), the insurance status, the history of hospitalization at psychiatric center, substance dependence, duration of disorder, related illness, and the type of treatment as independent variables.

\subsection{Ethical Considerations}

This study was approved by the ethics committee of Mazandaran University of Medical Sciences.

\subsection{Statistical Analysis}

Survival analysis is a statistical method used to study time to event data. One of the specific characteristics of survival analysis in comparison with other studies is censoring. Censorship occurs when we have incomplete information about the survival time of some subjects (12). In the present study, duration of hospital stay was considered as survival time variable, and the event was discharge from hospital. In the survival analysis there are various methods to fit the data. If the goal is to describe the survival regardless of covariates Non-parametric analysis methods such as the life table and Kaplan -Meier are used. However, if the goal is to investigate the effects of covariates on survival time, regression models are used. These models are divided into 2 main categories: proportional hazards $(\mathrm{PH})$ model and accelerated failure time (AFT) model. Proportional hazards regression model is used to obtain the effect of covariates on the risk function. In the accelerated regression model, the failure time of the effects of covariates on survival time logarithm will be obtained (13).

The (AFT) model is not used very often to analyze survival time data, however it suggests a statistical useful approach that is based on the survival curve rather than the hazard function. Many parametric models are AFT. Weibull is the most common distribution for parametric models in survival data. It should be noted that Weibull is the only model that can be used in both AFT and PH forms (14). This was checked for each variable by considering the parallel shape of the $\log (-\log )$ plots. The Kaplan-Meier estimates of lengths of stay across age groups of patients were plotted (Figure 1). Then, univariate tests conducted using the $\log$ rank test and variables with $\mathrm{P}<0.3$ were considered. Finally, Weibull regression analysis was performed to determine effective variables on the length of stay. Statistical analyses were performed using STATA 12 software and the value of $\alpha=0.05$ was considered significant. 


\section{Kaplan-Meier Survival Estimates}

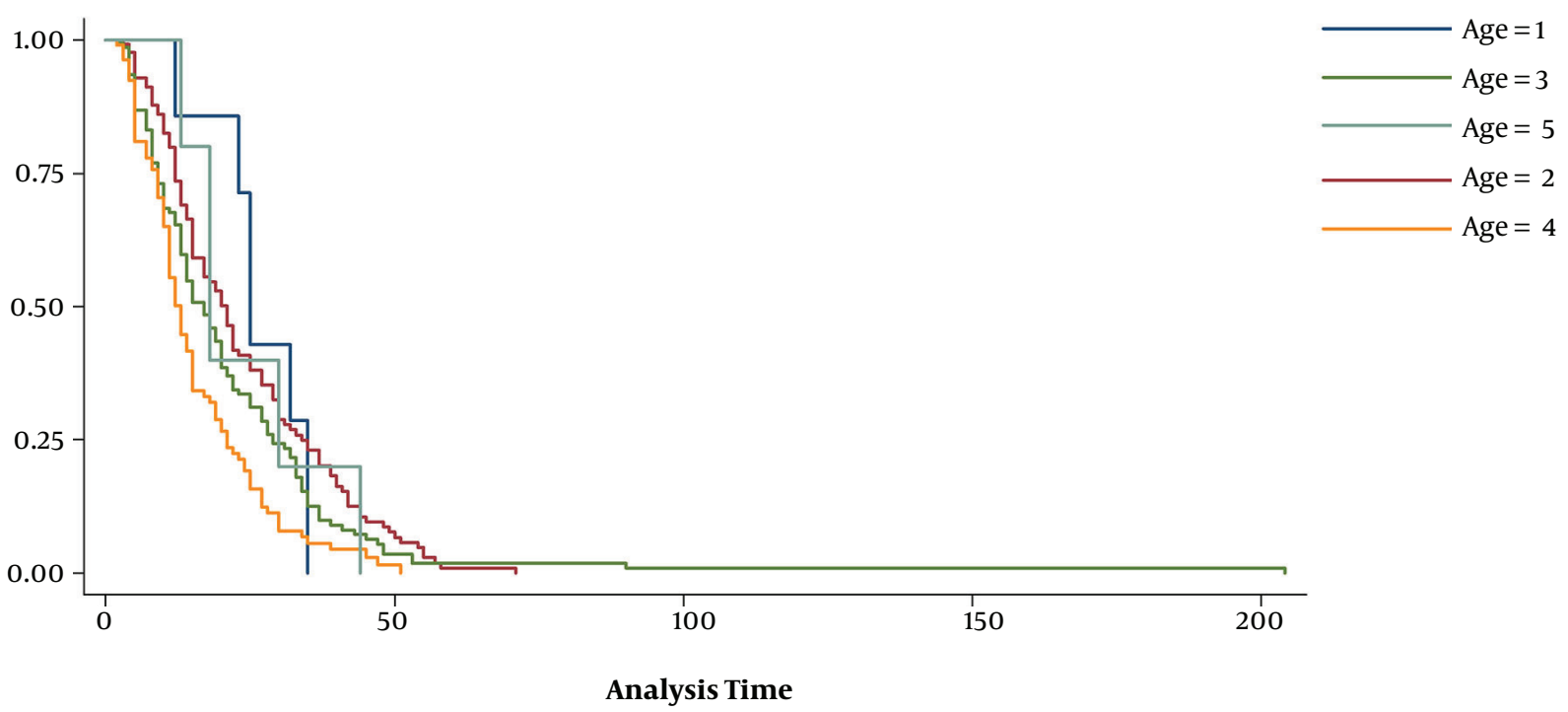

Figure 1. Kaplan-Meier Plot of Length of Stay Across Age Group of Patients

\section{Results}

Patients included in this study were 313 (73\%) males and 114 (27\%) females. The median length of hospital stay was 17 (interquartile range 10 - 29 day). The results showed that factors such as gender $(P=0.616)$, residence type $(P=0.6)$, insurance status $(\mathrm{P}=0.08)$, supplementary insurance $(\mathrm{P}=$ $0.07)$, substance dependence $(P=0.51)$, duration of disor$\operatorname{der}(\mathrm{P}=0.45)$, and related illness $(\mathrm{P}=0.78)$ were not associated with the duration of hospital stay. However age had a significant effect on the length of stay $(P=0.036)$. Also, patients who had received electroconvulsive therapy as well as occupational therapy, and patients with previous hospitalization had longer stay in hospital $(\mathrm{P}<0.001, \mathrm{P}=0.033$, respectively). The Weibull regression output is presented in Table 1.

\section{Discussion}

We applied statistical methods to make use of 'time to event' outcomes to identify factors affecting duration of stay in hospital in patients with anxiety disorders. As the results show aging has subtractive effect on the length of stay. According to estimates of the acceleration factor $(\gamma$ $=0.91$ ), the length of hospital stay in patients who were in the older age was $9 \%$ fewer than other patients. This result was the same as the duration of first time hospitalization in all psychiatric patients of the same hospital(15). Furthermore, in same study by Richter, the relation between the duration of hospitalization of mental patients, age, and type of diagnosis was evaluated at a hospital in Germany (16). The results showed that diagnosis and age consist of $5.1 \%$ of the variance of the length of stay in the hospital and $3.11 \%$ of the variance was determined by clinical and demographic characteristics. This finding doesn't support those of Cheng et al., who identified that older age is associated with the longest psychiatric inpatient length of stay (17). In the current study between factors such as gender, type of residence, insurance status, and duration of disorder to the duration of hospitalization, no relation was found. This result was different from the results of research conducted on psychiatric patients by Fakharian et al., at Razi Psychiatric hospital (18). In addition, patients with previous hospitalization had longer length of stay. Many patients with an anxiety disorder also have a co-occurring disorder or physical illness, which can affect the length of hospitalization and make recovery more difficult. In this study there was no significant association between related illness and the length of stay. We found that electroconvulsive therapy and occupational therapy were associated with increase in the length of stay. Considering the acceleration factor ( $\gamma=1.91)$ for electroconvulsive therapy and $(\gamma=1.79)$ for occupational therapy, patients who received these types of therapies are 91\% and 79\% more than other patients that were hospitalized.

Usually in the treatment of anxiety disorders, pharmacotherapy and psychotherapy are used, however, some anxiety disorders are treatment-resistant and do not im- 


\begin{tabular}{|c|c|c|c|c|c|c|}
\hline Variable & Coefficient & Std. Err. & $\mathbf{Z}$ & $\gamma$ & $\mathbf{P}>|\mathbf{z}|$ & 95\% Conf. Interval \\
\hline Gender & -0.04 & 0.08 & -0.5 & - & 0.616 & $0.81,1.42$ \\
\hline Age & -0.09 & 0.04 & -2.1 & 0.91 & 0.036 & $1.01,1.34$ \\
\hline Residence & 0.08 & 0.15 & 0.51 & - & 0.607 & $0.53,1.45$ \\
\hline Supplementary insurance & -0.14 & 0.08 & -1.77 & - & 0.076 & $0.97,1.65$ \\
\hline Previous hospitalization & 0.15 & 0.07 & 2.13 & 1.16 & 0.033 & $0.61,0.98$ \\
\hline Substance dependence & -0.04 & 0.06 & -0.66 & - & 0.51 & $0.87,1.32$ \\
\hline Duration of disorder & -0.003 & 0.004 & -0.75 & - & 0.453 & $0.99,1.02$ \\
\hline Related illness & -0.02 & 0.07 & -0.28 & - & 0.78 & $-0.17,0.12$ \\
\hline \multicolumn{7}{|l|}{ Type of treatment } \\
\hline \multicolumn{7}{|l|}{ Pharmacotherapy (baseline) } \\
\hline Psychotherapy & -0.45 & 0.08 & 0.77 & - & 0.442 & $0.29,15.8$ \\
\hline Electroconvulsive therapy & 0.64 & 0.09 & 6.42 & 1.91 & $<0.001$ & $0.23,0.47$ \\
\hline Occupational Therapy & 0.58 & 0.13 & 4.4 & 1.79 & $<0.001$ & $0.23,0.58$ \\
\hline
\end{tabular}

prove with these conventional therapies. Therefore, patients who receive electroconvulsive therapy and occupational therapy, despite long-term hospitalization, may benefit from sustainable therapeutic effects. Chand Rant examined reducing the duration of hospitalization in the psychiatric emergency unit and concluded that reducing the length of stay by 9 days to an average of 16 days did not increase in the readmission rate (19). Rasanesn examined changes in the treatment of schizophrenic by patients' gender and concluded that gender was not significantly related with the average duration of disease (20). In the current study, we found no significant relation between substance dependence and the length of hospitalization, However, Rise, in a study on schizophrenia patients who had simultaneously substance dependence disorder, concluded these patients compared to schizophrenic patients who had no substance dependence, had less hospitalization time, and suggest this hypothesis that the substance temporarily strengthen patients' symptoms and may also be effective on the prognosis of patients (21). In another study by Miller (2016), the relationship between active substance use at the time of psychiatric hospitalization and changes in suicide risk measures and length-of-stay was examined. The results showed patients with a substance use disorder had a 1.0 day shorter length-of stay and the lowest measures of suicide on admission (22).

Due to the high cost of health care, designing comprehensive programs will reduce at least $20 \%$ of health care costs. Therefore, in addition to appropriate health care, people can also benefit from financial justice in health care costs. Thus, considering the issue of reducing length of hospitalization in psychiatric patients would help to the economy of health system, reducing the burden of mental illnesses and more attention to the social factors of health care. The limitation of this study was the defective patient records due to inadequate software program. There may also be other causes that affect the length of hospital stay that are not considered in our study. Factors such as stay during the religious occasion months (Moharram, Zihajeh and Ramadan) and agriculture season, public attitudes to the mental patients, and private or public service delivery can be addressed in future researches.

\subsection{Conclusion}

In summary, we believe that the type of treatment is effective in reducing the duration of hospitalization. $\mathrm{Ag}$ ing has subtractive effect on the length of hospital stay. By identifying these relationships, we can make an effort to organize health care services. It seems that additional research concerning mental health care services may be required to identify more factors affecting the length of stay.

\section{Acknowledgments}

The authors express their gratitude to the participants of the study, the staff of psychiatric ward of Zare hospital, and also the student research committee of Mazandaran University of Medical Sciences. 


\section{Footnotes}

Authors' Contribution: Jamshid Yazdani-Charati and Soraya Mohammadi conceived and designed the evaluation. Mehran Zarghami helped to design the study. Soraya Mohammadi, Nadia Alipour, and Afsaneh Fendereski collected the clinical data. Jamshid Yazdani-Charati and Mehran Zarghami interpreted the clinical data. Soraya Mohammadi and Jamshid Yazdani-Charati performed the statistical analysis. Jamshid Yazdani-Charati, Mehran Zarghami, Soraya Mohammadi, Nadia Alipour, and Afsaneh Fendereski revised it critically for important intellectual content. All authors read and approved the final manuscript.

Declaration of Interest: None declared.

Funding/Support: This study was supported by Mazandaran University of Medical Sciences and the Student Research Committee (Ethical Code: 68).

\section{References}

1. World Health Organization. Investing in mental health. Department of Mental Health and Substance Dependence, Noncommunicable Diseases and Mental Health Gene; 2003-2008. Available from: http:// www.who.int/mental_health/en/investing_in_mnh_final.pdf.

2. Bootzin RR, Acocella JR. Abnormal psychology: Current perspectives. Crown Publishing Group/Random House; 1988.

3. Donald W, Goodwin MD. Anxiety. Ballantine Books. The United States of America; 1987.

4. Sookman D, Leahy RL. Treatment resistant anxiety disorders: Resolving impasses to symptom remission. New York: Taylor \& Francis; 2009.

5. Murray CJ, Vos T, Lozano R, Naghavi M, Flaxman AD, Michaud C, et al. Disability-adjusted life years (DALYs) for 291 diseases and injuries in 21 regions, 1990-2010: a systematic analysis for the Global Burden of Disease Study 2010. Lancet. 2012;380(9859):2197-223. doi: 10.1016/S01406736(12)61689-4. [PubMed: 23245608].

6. Ersoy K, Kavuncubasi S, Ozcan YA, Harris J2. Technical efficiencies of Turkish hospitals: DEA approach. J Med Syst. 1997;21(2):67-74. doi: 10.1023/A:1022801222540. [PubMed: 9297615].

7. Mills A. The economics of hospitals in developing countries. Part I: expenditure patterns. Health Policy Plan. 1990;5(2):107-17. doi: 10.1093/heapol/5.2.107.

8. Heartfield M. Regulating hospital use: length of stay, beds and whiteboards. Nurs Inq. 2005;12(1):21-6. doi: 10.1111/j.1440-1800.2005.00251.x. [PubMed: 15743439].
9. Atienza N, Garcia-Heras J, Munoz-Pichardo JM, Villa R. An application of mixture distributions in modelization of length of hospital stay. Stat Med. 2008;27(9):1403-20. doi: 10.1002/sim.3029. [PubMed: 17680551].

10. Borghans I, Heijink R, Kool T, Lagoe RJ, Westert GP. Benchmarking and reducing length of stay in Dutch hospitals. BMC Health Serv Res. 2008;8:220. doi:10.1186/1472-6963-8-220. [PubMed: 18950476].

11. Barnu H, Kutzin J. Public hospitals in developing countries. Baltimore MD: Johns Hopkin University pres; 1993. Available from: http: //documents.worldbank.org/curated/en/919871468740383421/ Public-hospitals-in-developing-countries-resource-use-costfinancing.

12. Croudace Andrew Beck Swaran Singh G TIM. Profiling activity in acute psychiatric services. J Ment Health. 2009;7(1):49-57. doi: $10.1080 / 09638239818337$.

13. Corporation S. Stata programming: reference manual. The United States of America: Stata Press; 2003.

14. Kieinbaum D. Survival Analysis, A Self, Learning text. 2nd ed. New York: Springer; 1996.

15. Nattaj AH,Zarghami M, Yazdani-Charati J, Vahedi M, Sheikholeslami A, Faghani Z. Factors associated with the length of first time hospitalization in a referral psychiatric hospital in North of Iran. Iran J Psychiatry Behav Sci. 2017;11(2). doi:10.5812/ijpbs.7579.

16. Richter D. [How significant is the comparison for length of stay in psychiatric hospitals based on diagnosis and age?]. Gesundheitswesen.1999;61(5):227-33. [PubMed: 10414016].

17. Cheng JE, Shumway M, Leary M, Mangurian CV. Patient Factors Associated with Extended Length of Stay in the Psychiatric Inpatient Units of a Large Urban County Hospital. Community Ment Health J. 2016;52(6):658-61. doi: 10.1007/s10597-015-9912-2. [PubMed: 26883829].

18. Fakharian F, Mazianani R, Fadaei F. [Study of the effective factors on lenght of stay among psychiatric patients in Razi Hospital]. Arch Rehab. 2002;3(3). Persian.

19. Edward-Chandran T, Malcolm DE, Bowen RC. Reduction of length of stay in an acute care psychiatric unit. Can JPsychiatry. 1996;41(1):49-51. doi: 10.1177/070674379604100111. [PubMed: 8919424].

20. Rasanen S, Hakko H, Herva A, Isohanni M, Nieminen P, Moring J. Community placement of long-stay psychiatric patients in northern Finland. Psychiatr Serv. 2000;51(3):383-5. doi: 10.1176/appi.ps.51.3.383. [PubMed: 10686249].

21. Ries RK, Russo J, Wingerson D, Snowden M, Comtois KA, Srebnik D, et al. Shorter hospital stays and more rapid improvement among patients with schizophrenia and substance disorders. Psychiatr Serv. 2000;51(2):210-5. doi: 10.1176/appi.ps.51.2.210. [PubMed: 10655005].

22. Miller KA, Hitschfeld MJ, Lineberry TW, Palmer BA. How does active substance use at psychiatric admission impact suicide risk and hospital length-of-stay? J Addict Dis. 2016;35(4):291-7. doi: 10.1080/10550887.2016.1177808. [PubMed: 27089003]. 